\title{
Исследование микроволнового поглощения в полупроводниках для устройств умножения частоты и управления выводом излучения непрерывных и импульсных гиротронов
}

\author{
(C) К.В. Маремьянин ${ }^{1}$, В.В. Паршин ${ }^{2}$, Е.А. Серов ${ }^{2}$, В.В. Румянцев ${ }^{1, \uparrow, ~ К . Е . ~ К у д р я в ц е в ~}{ }^{1}$, А.А. Дубинов ${ }^{1}$, \\ А.П. Фокин ${ }^{2}$, С.С. Морозов ${ }^{3}$, В.Я. Алешкин ${ }^{1}$, М.Ю. Глявин ${ }^{2}$, Г.Г. Денисов ${ }^{2}$, С.В. Морозов ${ }^{1}$
}

${ }^{1}$ Институт фризики микроструктур Российской академии наук, 603950 Нижний Новгород, Россия

${ }^{2}$ Институт прикладной физики Российской академии наук, 603950 Нижний Новгород, Россия

${ }^{3}$ Нижегородский государственный университет им. Лобачевского, 603950 Нижний Новгород, Россия

๑ E-mail: rumyantsev@ipmras.ru

Поступила в Редакцию 15 апреля 2020 г.

В окончательной редакции 21 апреля 2020 г.

Принята к публикации 21 апреля 2020 г.

\begin{abstract}
Приводятся результаты экспериментального исследования диэлектрических потерь в полупроводниковых кристаллах $\mathrm{GaAs}, \mathrm{InP}: \mathrm{Fe}$ и $\mathrm{Si}$ в миллиметровом диапазоне длин волн $(80-260$ ГГц) с использованием прецизионного оригинального метода измерений показателя преломления и тангенса угла диэлектрических потерь $\operatorname{tg} \delta$ на основе открытых высокодобротных резонаторов Фабри-Перо. Показано, что в сверхчистых полупроводниковых монокристаллических подложках GaAs потери в частотном диапазоне от 100 до 260 ГГц в основном определяются решеточным поглощением, в то время как в монокристаллическом кремнии основной механизм потерь - поглощение на свободных носителях заряда; при этом $\operatorname{tg} \delta \approx(1-2) \cdot 10^{-4}$ даже при заметной, на уровне $10^{12} \mathrm{~cm}^{-3}$, концентрации свободных носителей. В отличие от $\mathrm{GaAs}$ и $\mathrm{Si}$, в компенсированных кристаллах $\mathrm{InP}: \mathrm{Fe} \operatorname{tg} \delta$ практически не зависит от частоты в диапазоне от 100 до 260 ГГц, что связывается с проводимостью материала по прыжковому механизму. Полученные результаты могут быть использованы при проектировании и оптимизации микроволновых полупроводниковых приборов, в частности устройств умножения частоты и управляемого вывода излучения непрерывных и импульсных гиротронов.
\end{abstract}

Ключевые слова: субтерагерцовый диапазон, $\mathrm{GaAs}, \mathrm{InP}$ : Fe, $\mathrm{Si}$, поглощение, тангенс угла диэлектрических потерь.

DOI: $10.21883 /$ FTP.2020.09.49825.17

\section{1. Введение}

Мощные источники когерентного излучения дальнего инфракрасного (ДИК) и терагерцового (ТГц) диапазонов практически востребованы для решения многих задач фундаментального и прикладного характера, таких как развитие новых методов спектроскопии полупроводниковых структур и биологических объектов [1], управление химическими реакциями [2], манипулирование электронными состояниями в квантовых ямах [3] и др. Сложность создания подобных источников ДИК/ТГц диапазона связана с тем, что здесь плохо применимы подходы к построению излучающих устройств, разработанные для оптического или микроволнового диапазонов. В то же время для создания мощных источников излучения в указанных диапазонах (и последующего широкого использования на уровне лабораторий) можно было бы рассчитывать на нелинейное преобразование частоты излучения гиротронов [4], обеспечивающих генерацию в субтерагерцовом (суб-ТГц) и ТГц диапазонах с высокой выходной мощностью как в импульсном, так и в непрерывном режимах [5-7]. Развитие подобного подхода требует решения таких инженерно-технических задач, как разработка оптических ключей для управления выводом гиротронов и умножителей частоты для расширения рабочего частотного диапазона. Обе эти задачи могут быть потенциально решены с применением полупроводниковых материалов в качестве управляемых зеркал или нелинейных элементов. Отметим, что наряду с повышением частоты создание управляемых полупроводниковых зеркал открывает возможность реализации активных компрессоров, позволяющих обеспечить импульсы излучения наносекундной длительности с уровнем мощности в сотни МВт, что представляет интерес для задач высокоградиентного ускорения частиц [8]. Необходимость работы подобных устройств в мощном пучке гиротронного излучения определяет требование слабого поглощения микроволнового излучения используемыми материалами во избежание их перегрева и в конечном итоге механического разрушения.

Исследование диэлектрических потерь в различных полупроводниках материалах в миллиметровом и ТГц диапазонах представляется важным для развития соответствующей элементной базы для устройств и систем большой мощности. Несмотря на заметное число работ по данной тематике [9-17], имеющиеся в литературе 
Параметры исследуемых образцов и результаты измерений показателя преломления $\tilde{n}$ и тангенса угла потерь $\operatorname{tg} \delta$

\begin{tabular}{|c|c|c|c|}
\hline Материал & Частота $f$, ГГц & $\tilde{n}$ & $\operatorname{tg} \delta, 10^{-4}$ \\
\hline $\begin{array}{l}\text { Арсенид галлия }(\mathrm{GaAs}), \text { образец } 1, \\
R>10^{7} \text { Ом } \cdot \text { см }\end{array}$ & $\begin{array}{l}82.54 \\
165.08 \\
247.62\end{array}$ & $\begin{array}{l}3.588 \\
3.597 \\
3.589\end{array}$ & $\begin{array}{l}2.7 \\
3 \\
3.8\end{array}$ \\
\hline $\begin{array}{l}\text { Арсенид галлия }(\mathrm{GaAs}), \text { образец } 2, \\
R=(1.41-4.4) \cdot 10^{8} \text { Ом·см }\end{array}$ & $\begin{array}{l}123 \\
148 \\
172 \\
197\end{array}$ & $\begin{array}{l}3.592 \\
3.592 \\
3.592 \\
3.592\end{array}$ & $\begin{array}{l}2.8 \\
2.9 \\
3.1 \\
3.3\end{array}$ \\
\hline $\begin{array}{l}\text { Фосфид индия }(\mathrm{InP}: \mathrm{Fe}), \text { образец } 1, \\
R>10^{7} \text { Ом } \cdot \text { см }\end{array}$ & $\begin{array}{l}130.12 \\
195.17 \\
260.26\end{array}$ & $\begin{array}{l}3.527 \\
3.539 \\
3.532\end{array}$ & $\begin{array}{l}4.7 \\
4.6 \\
4.6\end{array}$ \\
\hline Фосфид индия (InP : Fe), образец 2 & $\begin{array}{l}200.80168 \\
194.71756 \\
188.64189 \\
182.5599 \\
176.46374 \\
170.38461 \\
164.29418 \\
158.21386 \\
152.13162 \\
146.0445 \\
139.95624 \\
133.87197 \\
127.78492 \\
121.70348 \\
115.61746 \\
109.53387\end{array}$ & $\begin{array}{l}3.534 \\
3.534 \\
3.534 \\
3.534 \\
3.533 \\
3.535 \\
3.534 \\
3.534 \\
3.533 \\
3.534 \\
3.534 \\
3.534 \\
3.534 \\
3.534 \\
3.535 \\
3.534\end{array}$ & $\begin{array}{l}4.5 \\
4.6 \\
4.5 \\
4.6 \\
4.6 \\
4.7 \\
4.7 \\
4.7 \\
4.85 \\
4.85 \\
4.8 \\
4.8 \\
4.8 \\
4.75 \\
4.8 \\
4.75\end{array}$ \\
\hline $\begin{array}{l}\text { Фосфид индия }(\mathrm{InP}: \mathrm{Fe}) \text {, образец } 3 \text {, } \\
R>5 \cdot 10^{6} \mathrm{OM} \cdot \mathrm{cm}\end{array}$ & $\begin{array}{l}85.82 \\
128.71 \\
171.63 \\
214.655\end{array}$ & $\begin{array}{c}3.525-3.528 \\
3.534-3.537 \\
3.530-3.533 \\
3.533\end{array}$ & $\begin{array}{l}4.8 \\
5 \\
4.8 \\
4.4\end{array}$ \\
\hline Фосфид индия (InP : Fe), образец 4 & $\begin{array}{l}109.530 \\
200.800\end{array}$ & $\begin{array}{l}3.534 \\
3.534\end{array}$ & $\begin{array}{l}4.7 \\
4.7\end{array}$ \\
\hline $\begin{array}{l}\text { Кремний }(\mathrm{Si}), \\
N \approx 6.5 \cdot 10^{11} \mathrm{~cm}^{-3}, n \text {-тип }\end{array}$ & $\begin{array}{l}135.9 \\
203.794\end{array}$ & $\begin{array}{l}3.415 \\
3.415\end{array}$ & $\begin{array}{l}1.7 \\
1.1\end{array}$ \\
\hline $\begin{array}{l}\text { Кремний }(\mathrm{Si}) \\
N \approx 2.2 \cdot 10^{12} \mathrm{~cm}^{-3}, p \text {-тип }\end{array}$ & 168.505 & 3.42 & 1.6 \\
\hline $\begin{array}{l}\text { Кремний }(\mathrm{Si}), \\
N \approx 2.3 \cdot 10^{12} \mathrm{~cm}^{-3}, n \text {-тип }\end{array}$ & 168.505 & 3.42 & 4.8 \\
\hline
\end{tabular}

данные представляются далеко не полными. В настоящей работе приводятся результаты измерения поглощения $(\operatorname{tg} \delta)$ ряда полупроводников, а именно $\mathrm{GaAs}, \mathrm{Si}$ и InP : Fe, в суб-ТГц области спектра и обсуждаются некоторые аспекты приборных применений указанных материалов в задачах умножения частоты и управления потоками мощного микроволнового излучения.

\section{2. Исследуемые образцы}

Интерес к высокочистым кристаллам GaAs и $\mathrm{Si}$ обусловлен возможностью построения на их основе оптически управляемых полупроводниковых зеркал для микроволнового излучения. „Включение“ такого зеркала можно осуществлять за счет „подсветки“ мощным межзонным излучением, формируя вблизи поверхности полупроводника отражающий слой с „квазиметаллической“ проводимостью вследствие высокой концентрации неравновесных носителей, $\sim\left(10^{18}-10^{19} \mathrm{~cm}^{-3}\right)$; подобная концепция была представлена на мегаваттном уровне мощности в ряде работ [18-20]. В „выключенном“ же состоянии (без подсветки) френелевское отражение от такого зеркала можно минимизировать за счет деструктивной интерференции волн, отраженных от передней 
и задней граней пластины. Критически важным здесь является вопрос исследования диэлектрических потерь и минимизации поглощения мощного суб-ТГц излучения в пластинах полупроводника, формирующих подобный „оптический ключ“".

Что касается InP, этот материал интересен благодаря большой величине квадратичной восприимчивости кристаллической решетки $\left(\chi^{(2)} \approx 3.45 \cdot 10^{-8} \mathrm{~cm} / \mathrm{B}\right.$; для сравнения в $\left.\mathrm{GaAs} \chi^{(2)} \approx 5.7 \cdot 10^{-9} \mathrm{~cm} / \mathrm{B}[21]\right)$. Таким образом, InP может быть использован в задачах умножения частоты гиротронного излучения для получения интенсивных ТГц и суб-ТГц пучков. В частности, в работе [22] показано, что эффективность нелинейного преобразования при генерации второй гармоники в кристаллах InP : Fe может достигать 3\%.

В данной работе исследованы пластины высокочистого GaAs с удельным сопротивлением $(R)$ не менее $10 \mathrm{MOM} \cdot$ см и пластины $\mathrm{Si}$ с удельным сопротивлением на уровне единиц кОм · см. Образцы кристаллов InP для снижения фоновой концентрации свободных носителей заряда были компенсированы железом (InP : Fe). Параметры исследуемых образцов приводятся в таблице.

\section{3. Методика измерений диэлектрических потерь}

В данной работе использован метод измерения сверхмалых потерь в диэлектриках с помощью высокодобротных открытых резонаторов Фабри-Перо [23-26]. Измеряемыми величинами для расчета показателя преломления и коэффициента диэлектрических потерь являются резонансные частоты и ширины резонансной кривой пустого и нагруженного резонатора. Измерения проводились на резонансных частотах анализируемых образцов, при которых по толщине пластины укладывается целое число полуволн. Расположение образца в резонаторе подбиралось таким образом, чтобы плоскости образца попадали в максимумы либо минимумы электрического поля стоячей волны в резонаторе, в соответствии с рекомендациями, сформулированными в работе [27]. Отметим, что в данной методике измерения показателя преломления $n$ и $\operatorname{tg} \delta$ плоскопараллельных пластин не требуется отдельного определения толщины пластин.

Источником излучения является лампа обратной волны с прецизионной цифровой системой управления частотой на основе фазовой автоподстройки частоты по гармонике синтезатора с опорным квантовым стандартом частоты, управляемого компьютером. Для регистрации формы резонансной кривой резонатора использовалась система быстрого сканирования по частоте $f$ в режиме фазовой автоподстройки с сохранением фазы излучения при переключениях. При характерной ширине резонанса $\sim 200$ кГц точность измерения ширины резонансной кривой после усреднения $\sim 500$ двухсторонних сканов достигала 20 Гц, что соответствовало погрешности измерения $\operatorname{tg} \delta$ на уровне $\sim\left(10^{-7}-10^{-6}\right)$. Это

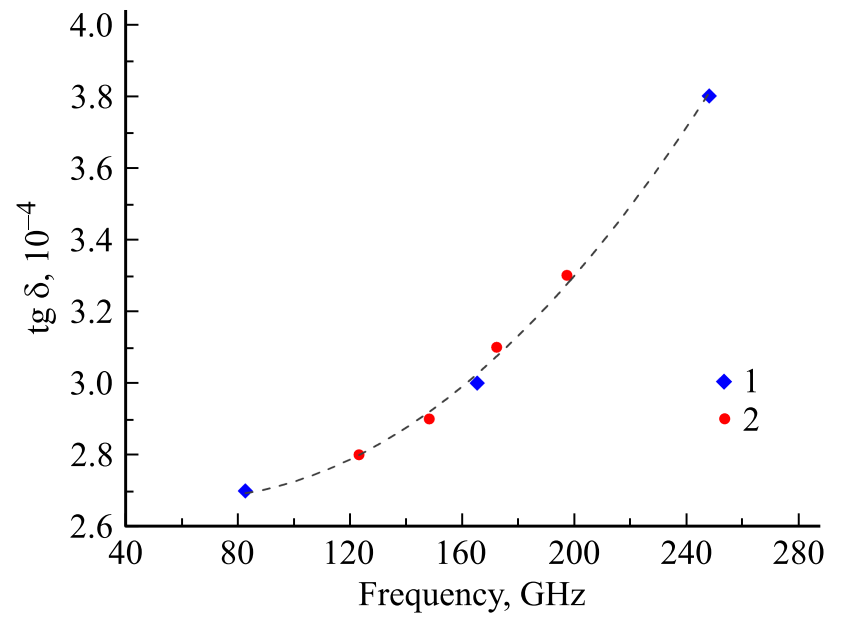

Рис. 1. Частотные зависимости тангенса угла потерь $\operatorname{tg} \delta$ для двух образцов полупроводниковых кристаллов GaAs.

более чем на порядок превосходит чувствительность спектрометров, построенных по другим схемам [28], и достаточно для исследования всех современных диэлектриков.

\section{4. Результаты и обсуждение}

\section{1. Измерение величины диэлектрических потерь в GaAs и Si}

Результаты измерений величины потерь (тангенса угла диэлектрических потерь $\operatorname{tg} \delta$ ) для рассмотренных образцов GaAs и $\mathrm{Si}$ приведены в таблице. Для пластин GaAs величина $\operatorname{tg} \delta$ растет с частотой (см. рис. 1); при этом данные, получаемые для различных образцов, хорошо укладываются на одну модельную кривую, соответствующую квадратичной зависимости потерь от частоты. Подобная частотная зависимость была предсказана в работе [9] для решеточного поглощения посредством „разностных“ двухфононных процессов: поглощение фотона с частотой $\omega$ сопровождается испусканием фонона с частотой $\omega_{1}$ и поглощением фонона с частотой $\omega_{2}=\omega_{1}-\omega$. Отметим, что помимо „квадратичной“ части потерь на рис. 1 видна также заметная „Подставка“ слабой зависимостью от частоты. Наличие подобного „фона“ можно связать с вкладом других разностных двухфононных процессов, для которых резонансная частота далека от исследуемого диапазона. Важно, что измеряемая величина потерь $(\operatorname{tg} \delta)$ в изучаемых пластинах GaAs не зависит от концентрации $n$ свободных носителей заряда (для достаточно чистых образцов с $\left.n<10^{8} \mathrm{~cm}^{-3}\right)$ и „решеточное“ поглощение действительно является основным (и принципиально не устраняемым) механизмом потерь в исследуемых образцах GaAs. 


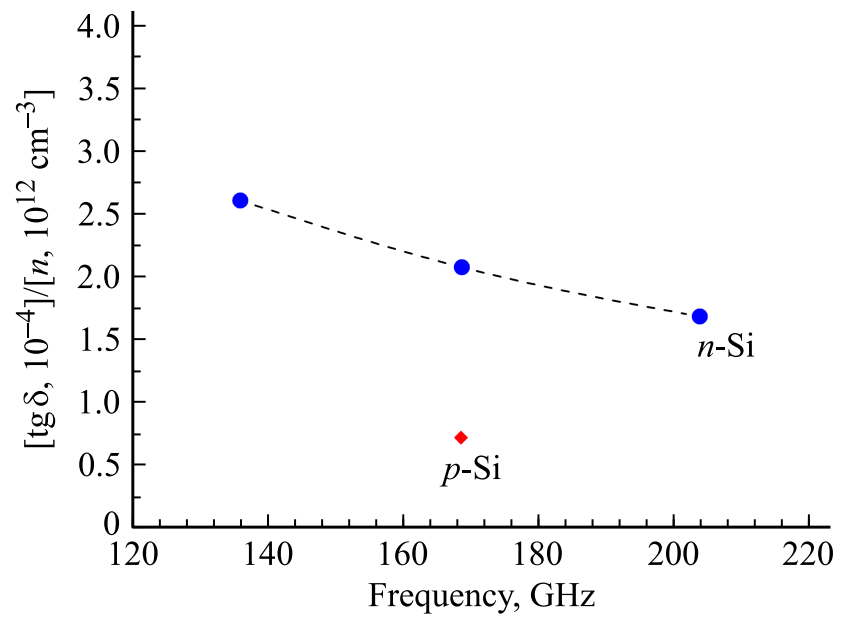

Рис. 2. Величина поглощения $(\operatorname{tg} \delta)$ для пластин кремния $n$ - и $p$-типа проводимости. Приведенные на рисунке данные нормированы на концентрацию свободных носителей заряда в изучаемых образцах (в ед. $10^{12} \mathrm{~cm}^{-3}$ ).

В таблице и на рис. 2 представлены также результаты исследований показателя преломления $n$ и величины $\operatorname{tg} \delta$ для нескольких образцов $c-\mathrm{Si}$. Видно, что величина потерь в кремнии в рассматриваемом частотном диапазоне заметно меньше, чем в арсениде галлия. Это объясняется тем, что структура кристаллической решетки кремния способствует подавлению многофононной „решеточной“ компоненты потерь; в этом случае основной вклад в поглощение материала в суб-ТГц диапазоне дают потери, связанные с проводимостью свободных носителей заряда. Такое предположение подтверждается как обратно пропорциональной зависимостью величины $\operatorname{tg} \delta$ от частоты пробного излучения, так и пропорциональным ростом потерь в образцах с увеличением концентрации свободных носителей заряда.

Несмотря на то что исследуемые образцы $\mathrm{Si}$ характеризуются относительно большой концентрацией остаточной примеси $\left(\sim 10^{12} \mathrm{~cm}^{-3}\right)$, получаемая величина потерь $(\operatorname{tg} \delta)$ заметно меньше, чем в высокочистых кристаллах GaAs. В сравнении с GaAs для кремния также характерны более высокая теплопроводность и в целом бо́льшая лучевая стойкость. Кроме того, меньший коэффициент поглощения на межзонных переходах в видимой и ближней ИК областях спектра позволяет реализовать при оптической накачке кремниевых пластин более однородный отражающий слой с высокой концентрацией неравновесных носителей заряда нужной толщины (на уровне десятков мкм). Отметим, что как в GaAs, так и в $\mathrm{Si}$ ширина запрещенной зоны является крайне „удобной“ для накачки доступными и практичными мощными твердотельными или полупроводниковыми лазерами - например, Nd : YAG. $\mathrm{C}$ этой точки зрения $\mathrm{GaAs}$ и $\mathrm{Si}$ значительно более практичны, чем алмаз, хотя для последнего характерен также весьма низкий уровень диэлектрических потерь. Некоторым преимуществом GaAs как основы для управляемых полупроводниковых зеркал (в сравнении с $\mathrm{Si}$ ) можно назвать достаточно быстрое „время переключения“ подобного зеркала за счет малого времени жизни ( 1 нс) избыточных носителей заряда в прямозонном полупроводнике, что потенциально позволяет формировать импульсы ТГц и суб-ТГц излучения наносекундной длительности. В случае же кремния для реализации столь быстродействующего оптического ключа необходимо будет существенно уменьшать (безызлучательное) время жизни неравновесных носителей, например, за счет легирования золотом. Известно, что легирование кремния золотом приведет к усилению рекомбинации по механизму Шокли-Рида-Холла и позволит сохранить низкий уровень потерь в суб-ТГц диапазоне $[10,17]$.

\section{2. Измерение величины диэлектрических потерь в InP : Fe}

Результаты измерения потерь $\operatorname{tg} \delta$ для образцов $\mathrm{InP}: \mathrm{Fe}$ приведены в таблице и на рис. 3. Ранее в работе [14] исследовались диэлектрические потери в кристаллах InP : Fe в области заметно бо́льших частот; наблюдаемые особенности связывались с решеточным поглощением, величина которого в целом была весьма существенной и на частотах $f>0.3$ ТГц приводила к $\operatorname{tg} \delta>10^{-3}$ (при этом величина $10^{-3}$ соответствовала погрешности измерений). Применение в данной работе более чувствительной методики измерений позволило аккуратно измерить величину диэлектрических потерь в диапазоне 100-260ГГц (рис. 3). Интересно, что для образцов InP : Fe величина $\operatorname{tg} \delta$ не имеет, в отличие от образцов GaAs, выраженной зависимости от частоты в диапазоне 100-260 ГГц и фактически представляет собой „плато“ в пределах $10 \%$.

В качестве гипотезы для объяснения наблюдаемого „Плато“ величины $\operatorname{tg} \delta$ можно было бы предложить следующую: в рассматриваемом диапазоне по мере уменьшения частоты излучения возрастает вклад поглощения на носителях заряда, что компенсирует спад

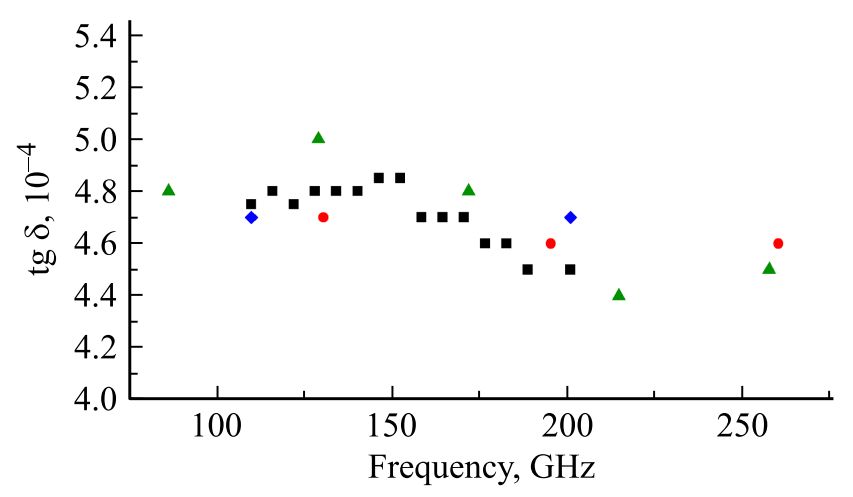

Рис. 3. Частотные зависимости тангенса угла потерь $\operatorname{tg} \delta$ для образцов компенсированного кристалла InP : Fe. 
величины „решеточного (фононного) поглощения в области меньших частот. Действительно, полученные в данной работе значения $\operatorname{tg} \delta$ на частотах 250-260ГГц более чем в 2 раза ниже приводимых в [14] и, таким образом, хорошо укладываются на длинноволновое плечо линии поглощения, связываемое с разностными фононными процессами LO-TO (между продольными и поперечными оптическими фононами). В то же время при фиксированной величине проводимости $\sigma=e n \mu$ (которая для исследуемых образцов составляет не более $\left.10^{-6}(\mathrm{Oм} \cdot \mathrm{cm})^{-1}\right)$, где $n$ и $\mu-$ соответственно концентрация и подвижность электронов в InP, оценка величины поглощения на свободных носителях заряда из соотношения

$$
\operatorname{tg} \delta=\frac{\operatorname{Im} \varepsilon}{\operatorname{Re} \varepsilon} \sim \frac{\gamma_{p}}{\omega} \frac{\omega_{p}^{2}}{\omega^{2}+\gamma_{p}^{2}},
$$

где $\gamma_{p}=e /\left(m^{*} \mu\right)$ и $\omega_{p}=4 \pi n e^{2} /\left(m^{*} \varepsilon_{\infty}\right), e-$ заряд электрона, $m^{*}=0.08 m_{0}-$ эффективная масса, $\varepsilon_{\infty} \approx 9.5-$ высокочастотная диэлектрическая проницаемость InP, дает величину потерь, не превышающую $\operatorname{tg} \delta \sim 10^{-5}$. Согласно такой оценке, поглощение на свободных носителях заряда является слишком слабым и может определять лишь небольшой разброс в измеряемых величинах $\operatorname{tg} \delta$ для разных образцов. В качестве альтернативы можно предложить потери на локализованных носителях заряда, возникающие за счет проводимости образца по прыжковому механизму. Такая возможность упоминается в работе [14] и наблюдалась в работе [29], а сублинейный характер зависимости прыжковой проводимости от частоты $\left(\sigma \propto \omega^{v}, v \leq 1\right.$ [30], $v \approx 0.7-0.8$ в работе [14]) позволяет объяснить наблюдаемый слабый рост величины потерь с частотой: $\operatorname{tg} \delta \propto \omega^{(0.7-0.8)} / \omega$.

В целом низкие потери и практически нулевая дисперсия показателя преломления InP : Fe в исследованном спектральном диапазоне позволяют рассчитывать при умножении частоты на длину когерентности $\sim(5-10)$ см. В этом случае эффективность нелинейного преобразования может достигать нескольких процентов при достаточно умеренных значениях интенсивности излучения $\left(\sim 50 \mathrm{\kappa BT} / \mathrm{cm}^{2}\right)$ на фундаментальной частоте.

\section{5. Заключение}

В работе выполнены измерения диэлектрических потерь в полупроводниковых кристаллах GaAs, InP : Fe и $\mathrm{Si}$ в частотном диапазоне 100-260 ГГц. Показано, что в высокочистых кристаллах GaAs основная величина потерь определяется решеточным поглощением по „разностному“ двухфононному механизму. В высокочистом кремнии фононное поглощение существенно подавлено и основным механизмом потерь является поглощение на свободных носителях по механизму Друде, а общая величина потерь может быть весьма малой: $\operatorname{tg} \delta \approx(1-2) \cdot 10^{-4}$ даже при концентрации свободных носителей $\sim 10^{12} \mathrm{~cm}^{-3}$.

Для кристаллов InP : Fe измеренные величины потерь заметно превышают оценочные значения как для решеточного поглощения, так и для поглощения на свободных носителях заряда. Высказано предположение, что заметный вклад в поглощение микроволнового излучения в кристаллах InP: Fe может быть связан с проводимостью по прыжковому механизму. Полученные данные могут быть полезны при проектировании и оптимизации микроволновых и терагерцовых полупроводниковых приборов на основе GaAs, InP : Fe и Si.

\section{Финансирование работы}

Работа выполнена при финансовой поддержке Российского научного фонда, грант 18-79-10112. Постановка задачи об использовании полупроводниковых зеркал в схеме сжатия импульсов мегаваттных гиротронов для высокоградиентного ускорения частиц и оценка основных параметров полупроводников, требуемых для решения этой задачи, сформулирована в гранте Российского научного фонда 19-79-30071.

\section{Конфликт интересов}

Авторы заявляют, что у них нет конфликта интересов.

\section{Список литературы}

[1] S.W. Smye, J.M. Chamberlain, A.J. Fitzgerald, E. Berry. Phys. Med. Biol., 46 (9), R101 (2001).

[2] N. Dudovich, D. Oron, Y. Silberberg. Nature, 418 (6897), 512 (2002).

[3] B.E. Cole, J.B. Williams, B.T. King, M.S. Sherwin, C.R. Stanley. Nature, 410 (6824), 60 (2001).

[4] A.V. Gaponov, M.I. Petelin, V.K. Yulpatov. Radiophys. Quant. Electron., 10 (9-10), 794 (1971).

[5] M. Thumm. J. Infrared, Millimeter, Terahertz Waves, 41 (1), 1 (2020).

[6] M.Y. Glyavin, G.G. Denisov, V.E. Zapevalov, M.A. Koshelev, M.Y. Tretyakov, A.I. Tsvetkov. Physics-Uspekhi, 59 (6), 595 (2016).

[7] T. Idehara, S.P. Sabchevski, M. Glyavin, S. Mitsudo. Appl. Sci., 10 (3), 980 (2020).

[8] E.A. Nanni, W.R. Huang, K.-H. Hong, K. Ravi, A. Fallahi, G. Moriena, R.J. Dwayne Miller, F.X. Kärtner. Nature Commun., 6, 8486 (2015).

[9] B.M. Garin. ICMWFST'96, 4th Int. Conf. on Millimeter Wave and Far Infrared Science and Technology, Proc. (Beijing, China, 1996) p. 311.

[10] B.A. Andreev, T.V. Kotereva, V.V. Parshin, V.B. Shmagin, R. Heidinger. Inorg. Mater., 33 (11), 1100 (1997).

[11] J.A. Hejase, P.R. Paladhi, P.P. Chahal. IEEE Trans. Components, Packaging, Manufacturing Technol., 1 (11), 1685 (2011).

[12] J. Krupka, J. Breeze, A. Centeno, N. Alford, T. Claussen, L. Jensen. IEEE Trans. Microwave Theory and Techniques, 54 (11), 3995 (2006). 
[13] J. Krupka, J.G. Hartnett, M. Piersa. Appl. Phys. Lett., 98 (11), 112112 (2011).

[14] L.N. Alyabyeva, E.S. Zhukova, M.A. Belkin, B.P. Gorshunov. Sci. Rep., 7 (1), 7360 (2017).

[15] B.M. Garin, V.V. Parshin, S.E. Myasnikova, V.G. Ralchenko. Diamond Relat. Mater., 12 (10-11), 1755 (2003).

[16] R. Golovashchenko, V. Derkach, S. Tarapov. Radiofizika I Elektronika, 20 (4), 31 (2015).

[17] J. Molla, R. Vila, R. Heidinger, A. Ibarra. J. Nucl. Mater., 258-263, 1884 (1998).

[18] M. Kulygin, G. Denisov. J. Infrared, Millimeter, Terahertz Waves, 33 (6), 638 (2012).

[19] J.F. Picard, S.C. Schaub, G. Rosenzweig, J.C. Stephens, M.A. Shapiro, R.J. Temkin. Appl. Phys. Lett., 114 (16), 164102 (2019).

[20] A.A. Vikharev, G.G. Denisov, V.V. Kocharovsky, S.V. Kuzikov, V.V. Parshin, N.Y. Peskov, A.N. Stepanov, D.I. Sobolev, M.Y. Shmelev. Radiophys. Quant. Electron., 50 (10), 786 (2007).

[21] O. Madelung. Semiconductors: Data Handbook (N.Y., Springer Verlag, 2003).

[22] V.V. Rumyantsev, K.V. Maremyanin, A.P. Fokin, A.A. Dubinov, V.V. Utochkin, M.Y. Glyavin, N.N. Mikhailov, S.A. Dvoretskii, S.V. Morozov, V.I. Gavrilenko. Semiconductors, 53 (9), 1217 (2019).

[23] Y.A. Dryagin, V.V. Parshin. Int. J. Infrared and Millimeter Waves, 13 (7), 1023 (1992).

[24] A.F. Krupnov, M.Y. Tretyakov, V.V. Parshin, V.N. Shanin, M.I. Kirillov. Int. J. Infrared and Millimeter Waves, 20 (10), 1731 (1999).

[25] V.V. Parshin, M.Y. Tretyakov, M.A. Koshelev, E.A. Serov. Radiophys. Quant. Electron., 52 (8), 525 (2010).

[26] V.N. Shanin, V.V. Dorovskikh, M.Y. Tretyakov, V.V. Parshin, A.P. Shkaev. Instrum. Exp. Tech., 46 (6), 798 (2003).

[27] E.V. Koposova, S.E. Myasnikova, V.V. Parshin, S.N. Vlasov. Diamond Relat. Mater., 11 (8), 1485 (2002).

[28] A.F. Krupnov, M.Y. Tretyakov, V.V. Parshin, V.N. Shanin, S.E. Myasnikova. J. Mol. Spectr., 202 (1), 107 (2000).

[29] N.F. Mott, E.A. Davis. Electronic Processes in Noncrystalline Solids (London, Oxford University, 1971).

[30] K. Khirouni, H. Maaref, J.C. Bourgoin, J.C. Garcia. Mater. Sci. Engin. B, 22, 86 (1993).

Редактор Л.В. Шаронова

\section{Studies of microwave absorption in semiconductors for frequency multiplication devices and radiation output control of continuous and pulsed gyrotrons}

K.V. Maremyanin ${ }^{1}$, V.V. Parshin ${ }^{2}$, E.A. Serov ${ }^{2}$, V.V. Rumyantsev ${ }^{1}$, K.E. Kudryavtsev ${ }^{1}$, A.A. Dubinov', A.P. Fokin ${ }^{2}$, S.S. Morozov ${ }^{3}$, V.Ya. Aleshkin ${ }^{1}$, M.Yu. Glyavin $^{2}$, G.G. Denisov' ${ }^{2}$, S.V. Morozov ${ }^{1}$

${ }^{1}$ Institute for Physics of Microstructures, Russian Academy of Sciences, 603950 Nizhny Novgorod, Russia

${ }^{2}$ Institute of Applied Physics, Russian Academy of Sciences, 603950 Nizhny Novgorod, Russia ${ }^{3}$ Lobachevsky State University of Nizhny Novgorod, 603950 Nizhny Novgorod, Russia

Abstract The paper presents the results of an experimental study of dielectric losses in $\mathrm{GaAs}, \mathrm{InP}: \mathrm{Fe}$ and $\mathrm{Si}$ semiconductor crystals in the millimeter wavelength range $(80-260 \mathrm{GHz})$ using a precision original method for measuring the refractive index and dielectric loss tangent $\operatorname{tg} \delta$ based on open high-Q Fabry-Perot resonators. It is shown that in ultrapure semiconductor singlecrystal GaAs substrates, losses in the frequency range from 100 to $260 \mathrm{GHz}$ are mainly determined by lattice absorption, while in single-crystal silicon, the main loss mechanism is absorption due to free charge carriers. In the latter case, the loss tangent is $\operatorname{tg} \delta \approx(1-2) \cdot 10^{-4}$ even for a noticeable (at the level of $10^{12} \mathrm{~cm}^{-3}$ ) concentration of free carriers. In contrast to $\mathrm{GaAs}$ and $\mathrm{Si}$, in compensated $\mathrm{InP}: \mathrm{Fe} \operatorname{tg} \delta$ does not change significantly with the frequency in the range from 100 to $260 \mathrm{GHz}$, which is associated with the hopping mechanism of material conductivity. The results can be used for the design and optimization of microwave semiconductor devices, in particular, frequency multiplication devices and controlled output of radiation from continuous and pulsed gyrotrons. 